\title{
Original
}

\section{Cyclophosphamide Promotes Arrested Development of the Dental Root in Mice}

\author{
Satoru Matsuo $^{1,2)}$, Masako Toda-Nakamura ${ }^{1)}$, Kyoko Oka ${ }^{1,3)}$, Hiroshi Kajiya ${ }^{2,3,4)}$, Kayoko Ogata ${ }^{3,5)}$, Hanako Ishii ${ }^{1,2)}$, Masao Ozaki ${ }^{1)}$ and \\ Jun Ohno ${ }^{2,3)}$
}

${ }^{1)}$ Section of Pediatric Dentistry, Department of Oral Growth and Development, Fukuoka Dental College, Fukuoka, Japan

${ }^{2)}$ Research Center for Regenerative Medicine, Fukuoka Dental College, Fukuoka, Japan

3) Oral Medicine Research Center, Fukuoka Dental College, Fukuoka, Japan

4) Section of Cellular Physiology, Department of Physiological Science and Molecular Biology, Fukuoka Dental College, Fukuoka, Japan

${ }^{5)}$ Section of Functional Structure, Department of Morphological Biology, Fukuoka Dental College, Fukuoka, Japan

(Accepted for publication, January 20, 2020)

\begin{abstract}
Cyclophosphamide (CPA) is a commonly used chemotherapeutic agent to treat cancer. Among its many side effects is the well-known consequence on tooth development when administered at early ages. This study elucidated the effects of CPA on development of the mandibular molar in mice. Mice received a single intraperitoneal injection of CPA at different doses and development times. CPA treatment led to weight loss and alopecia but had no effect on disturbances in tooth eruption or crown shape. However, at higher doses, there was arrested root development and early apical foramen closure histologically related to the formation of the cervical loop structure in the apical portion of the root. In cell culture experiments, the Hertwig's epithelial root sheath cell line (HERS01a) was cultured with or without CPA. At high doses of CPA, HERS01a cells showed decreases in E-cadherin expression, while N-cadherin expression was upregulated, indicating that this cadherin switch may promote an epithelial-to-mesenchymal transition (EMT)-like phenomenon. These findings suggest that administration of high doses of CPA can lead to arrested root development of the molars and an EMT-like phenomenon.
\end{abstract}

Key words: Tooth root, Cyclophosphamide, Hertwig’s epithelial root sheath, Epithelial-to-mesenchymal transition

\section{Introduction}

The overall mortality rates of childhood malignant tumors have decreased to approximately $20 \%$ due to notable improvements in therapies and early diagnosis ${ }^{1,2)}$. Chemotherapy medicines are widely used and effective in the treatment of childhood malignant tumors ${ }^{3)}$. Chemotherapeutic agents are expected to target malignant cells specifically without affecting healthy tissue, but there still are many off-target effects of cytotoxic treatment. Due to its capacity to interfere with cancer cell division, the N-mustard derivative alkylating drug cyclophosphamide (CPA) is widely used in the treatment of cancer.

Despite improving survivorship of children with malignant tumors, the long-term effects of chemotherapy also must be considered ${ }^{4,5)}$. Children who receive chemotherapy before the age of five can represent extensive damage to their dentition, including certain dental anomalies, such as hypodontia, microdontia, enamel hypoplasia, dental caries, and atypical crown or root formation ${ }^{6,7)}$. These dental anomalies may lead to anatomic, functional, and aesthetic problems, and severe abnormalities can cause malocclusion that can affect facial development and impacts quality of life ${ }^{6}$. However, it is difficult to attribute these effects to any single or treatment modality, as multimodal therapy is employed in almost all childhood malignant tumors ${ }^{8)}$.

Disturbances in tooth eruption can severely impact the oral health quality of life for children receiving long-term chemotherapy ${ }^{9)}$. Root

Correspondence to: Dr. Jun Ohno, Research Center for Regenerative Medicine, Fukuoka Dental College, 2-15-1 Tamura, Sawara-ku, Fukuoka, Fukuoka 8140193, Japan; Tel: +81928010411 (Ext 684); Fax: +81928014909; E-mail: johno@college.fdcnet.ac.jp formation, which causes an overall increase in tooth length, accompanies intraosseous eruption during the emergence process. Therefore, atypical root formation induced by antineoplastic therapy may lead to disturbances in tooth eruption. Among these problems with root formation, foreshortening of roots elicited by premature closure of apices results directly in decreased tooth length and leads to impaired tooth eruption. However, it has been shown that rootless teeth can erupt into the mouth ${ }^{10-13)}$. The disruption of root formation and tooth eruption is caused by irradiation of the mouth ${ }^{12,14,15)}$. This conflicting evidences prompted us to investigate possible interactions between root formation and tooth eruption.

Hertwig's epithelial root sheath (HERS) plays a role in controlling root and cementum formation. During the development of the tooth root, which starts after crown formation when the enamel tissue has reached the future cementoenamel junction, the HERS arises from an elongated apical region of the enamel organ and consists of a bilayer epithelial structure between the dental papilla and dental follicle ${ }^{16}$. The HERS then grows apically and guides root formation, determining the size, shape, and number of the tooth's roots ${ }^{17)}$. During this continuous movement of HERS toward the apical portion of the tooth, the HERS gives rise to a mesh network via localized apoptosis or epithelial-to-mesenchymal transition (EMT). This network facilitates the interaction between epithelial cells from the HERS and cranial neural crest-derived mesenchymal cells to develop the formation of cementum $^{18,19)}$. Therefore, disturbances in the function of the HERS cells lead to impairment in tooth root structure, shape, number, and length as well as cementogenesis ${ }^{20)}$. Molecular mechanisms leading to the dysregulated 
function of HERS cells remain unclear. Thus, this study aims to determine whether CPA administration influences root development of the mandibular molar in the mice. In addition, we investigated the effects of CPA on characterization of HERS cells in vitro.

\section{Materials and methods Experimental design and tissue preparation}

The study protocol of all animal experiments was approved by the Animal Care and Use Committee of Fukuoka Dental College, Fukuoka, Japan (approval no. 17021). Nineteen day-pregnant ICR mice were purchased from KBT Oriental Co., LTD (Tosu, Japan). After birth, the mice were housed with their mothers and provided free access to breast milk, and they had access to standard solid mouse feed and distilled water ad libitum after weaning.

At 3, 6, and 9 days of age (postnatal (PN) 3, PN 6, and PN 9), mice $(\mathrm{n}=120)$ received a single intraperitoneal injection $(100 \mu \mathrm{l})$ of CPA (Endoxan, Shionogi \& Co., Ltd., Tokyo, Japan) at a dose of 50, 100, and $200 \mathrm{mg} / \mathrm{kg}$ body weight. As a control group, additional mice were intraperitoneally injected with $100 \mu \mathrm{l}$ of saline. Body weight and appearance were observed daily throughout the experimental period.

At PN14, 21, and 28, mice treated with or without CPA were euthanatized by intraperitoneal injection of an overdose of anesthetic agents, and their mandibles were removed and dissected.

\section{Histological analysis}

Following autopsy at each time point, unilateral removed mandibles were fixed in $4 \%$ paraformaldehyde in phosphate buffered saline, decalcified in $10 \%$ formic acid for 2 weeks at $4^{\circ} \mathrm{C}$ and were then embedded in paraffin. The paraffin-embedded specimens were sagittally cut and serial sections were stained with hematoxylin and eosin (H\&E) to visualize any histological changes.

\section{Micro-computed tomography (micro-CT) analysis}

Fixed mandibles were scanned with a micro-CT system (Skyscan-1176 micro-CT; Bruker microCT, Kontich, Belgium) operated at $50 \mathrm{kVp}$ and $500 \mathrm{~mA}$. The scan size of each image was approximately 9 $\mathrm{mm}$. The anatomical length of the medial root of each molar was measured along the medial margin of the tooth from the cementoenamel junction to the root apex using three-dimensional (3D) analytic software (WinROOF, MITANI, Tokyo, Japan). The sizes of the orifice were observed from the apical side and assessed by the inner margin of the apex foramina of the medial roots as apex foramen area using Image J 1.46r software (National Institutes of Health (NIH), Bethesda, MD, USA).

\section{Cell culture and CPA treatment of HERS cells}

The HERS cell line, HERS01a, was previously established ${ }^{21)}$. HERS01a cells were cultured in Dulbecco's Modified Eagle Medium: Nutrient Mixture F-12 (Gibco, Grand Island, NY, USA) supplemented with B27 (Invitrogen, Carlsbad, CA, USA), fibroblast growth factor-2 (FGF2, 10 ng/ml, Fiblast Spray, Kaken Pharmaceutical Co., Ltd., Tokyo, Japan), epidermal growth factor (EGF, $10 \mathrm{ng} / \mathrm{ml}$, Pepro Tech, Rocky Hill, NJ, USA), and penicillin-streptomycin $(100 \mathrm{U} / \mathrm{ml})$ in a humidified atmosphere of $5 \% \mathrm{CO}_{2}$ at $37^{\circ} \mathrm{C}$. CPA $(0.18,0.36$, and $0.72 \mu \mathrm{M})$ was added to the culture medium of HERS01a cells in $60-\mathrm{mm}$ dishes or 10 -well culture slides.

\section{Cell viability assay}

To determine CPA sensitivities, cell viability was performed using a trypan blue exclusion test. Briefly, HERS01a cells were washed with phosphate-buffered saline (PBS), harvested from the dishes via trypsinization, resuspended in PBS, and diluted $1: 1$ in $0.4 \%$ trypan blue solution. Cell viability was calculated using a Contest Automated Cell Counter (Invitrogen) following the manufacturer's instructions.

\section{Western blot analysis}

The cells were lysed in Cell Lysis Buffer (Cell Signaling Technology, Danvers, MA, USA) containing a 1x Protease/Phosphatase Inhibitor Cocktail (Cell Signaling Technology). The protein content was measured with a protein assay kit (Pierce, Hercules, CA, USA). Protein samples, together with a protein marker (Precision Plus Protein Western C Standards; Bio-Rad, Richmond, CA, USA), were separated on $12 \%$ Mini-Protean TGX gels (Bio-Rad) for $30 \mathrm{~min}$ at $200 \mathrm{~V}$. The separated gels were transferred to a polyvinylidene fluoride membrane for $3 \mathrm{~min}$ using the Trans-Blot Turbo Transfer system (Bio-Rad) with Trans-Blot Transfer Packs. Western blots were processed on the iBind Western System (Life Technologies, Carlsbad, CA, USA) with anti- $\beta$-actin (Bio-Rad) ,anti-E-cadherin (1:1000; Cell Signaling Technology) and anti-N-cadherin (1:1000; Abcam, Cambridge, UK and horseradish peroxidase-conjugated anti-rabbit secondary antibodies (Bio-Rad) . The protein bands were developed using an enhanced chemiluminescence system (SignalFire Plus ECL Reagent, Cell Signal Technology). Band density was quantified using the NIH ImageJ software.

\section{Immunocytochemical analysis}

HERS01a cells treated with or without CPA were cultured on the 10well slides and fixed with $4 \%$ paraformaldehyde for 10 min and washed in $0.3 \%$ Triton-X in PBS for 15 min. The cells were incubated with anti-E-cadherin (1:200; Cell Signaling Technology) and anti-N-cadherin (1:200; Abcam), at $4{ }^{\circ} \mathrm{C}$ overnight. After washing with PBS, the cells were incubated with anti-rabbit IgG or anti-mouse IgG conjugated with Alexa Fluor 488 (1:200; Molecular Probes, Eugene, OR, USA) at room temperature for $45 \mathrm{~min}$. To visualize the nuclei, the cells were counterstained with 4, 6-diamidino-2-phenylindole (DAPI; Vector Laboratories, Inc., Burlingame, CA, USA).

\section{Statistical analysis}

Statistical analyses were performed with one-way analysis of variance (ANOVA), and Bonferroni's multiple comparison test to determine the statistical differences among the samples. Data are presented as the mean \pm standard deviation (SD) and $\mathrm{p}$ values $<0.05$ were considered to be statistically significant.

\section{Results \\ Systemic effects of CPA treatment in mice}

We first examined systemic effects of CPA in mice. Body size from the experimental groups was smaller than that from the control group at each time point (Fig. 1A). Mice treated with $200 \mathrm{mg} / \mathrm{kg}$ CPA were markedly smaller, compared to the other groups, after PN 21. In the 200 $\mathrm{mg} / \mathrm{kg}$ CPA-treated group, there a depression of hair growth that started at PN 14, resulting in focal hair loss in the body, compared to the other groups (Fig. 1A). After PN 21, rapid alopecia developed in mice treated with $200 \mathrm{mg} / \mathrm{kg} \mathrm{CPA}$, but mice treated with 50 or $100 \mathrm{mg} \mathrm{CPA} / \mathrm{kg}$ showed no hair loss. Furthermore, the $200 \mathrm{mg} / \mathrm{kg}$ group led to gradual weight loss at PN 14 and 21 (Fig. 1B). At PN 28, significant weight loss was observed in mice treated with $200 \mathrm{mg} / \mathrm{kg}(53.7 \pm 0.14 \%$ vs. the control, $\mathrm{p}<0.001$ ), whereas mice treated with doses below $200 \mathrm{mg} / \mathrm{kg}$ were not effective at countering CPA-induced weight loss. These findings indicate that a dose of $200 \mathrm{mg} / \mathrm{kg}$ CPA-induced systemic changes, includ- 
A

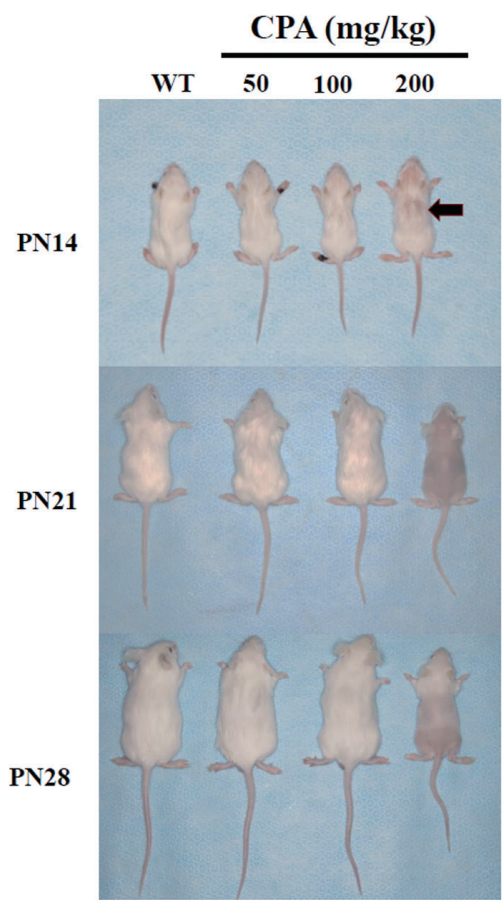

B

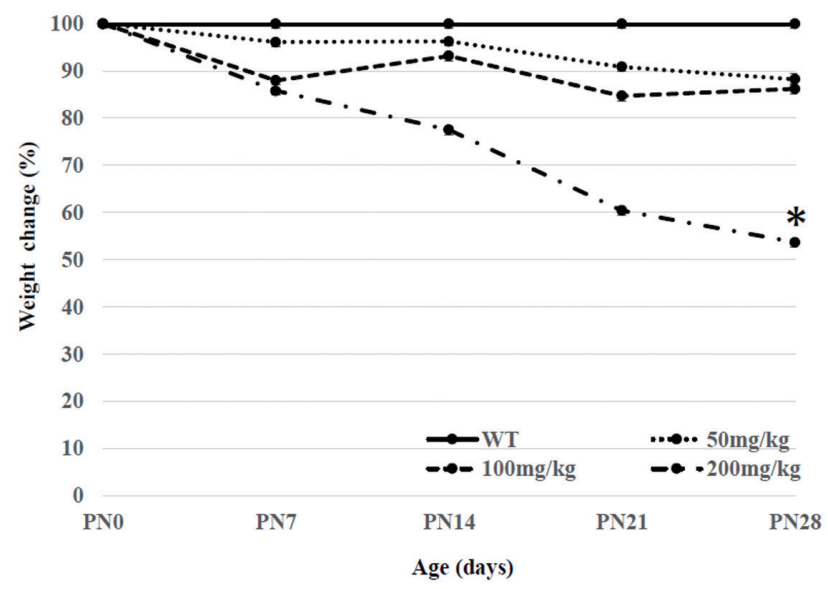

Figure 1. Macroscopic effects of CPA treatment in ICR mice. A: Photographs of mice treated with or without CPA at PN 14, 21, and 28. CPA-related severity of $200 \mathrm{mg} / \mathrm{kg}$ CPA-treated mice can be seen at PN 21 and 28. WT: wild type (control). Arrow, focal hair loss. B: Changes in body weight (\%) shown as a fraction of body weight in each experimental group and control at PN 14, 21, and 28. The values in the lines are presented as mean $\pm \mathrm{SD} .{ }^{*} \mathrm{p}<0.01$ vs. the control group.

\section{$\mathrm{CPA}(\mathrm{mg} / \mathrm{kg})$}

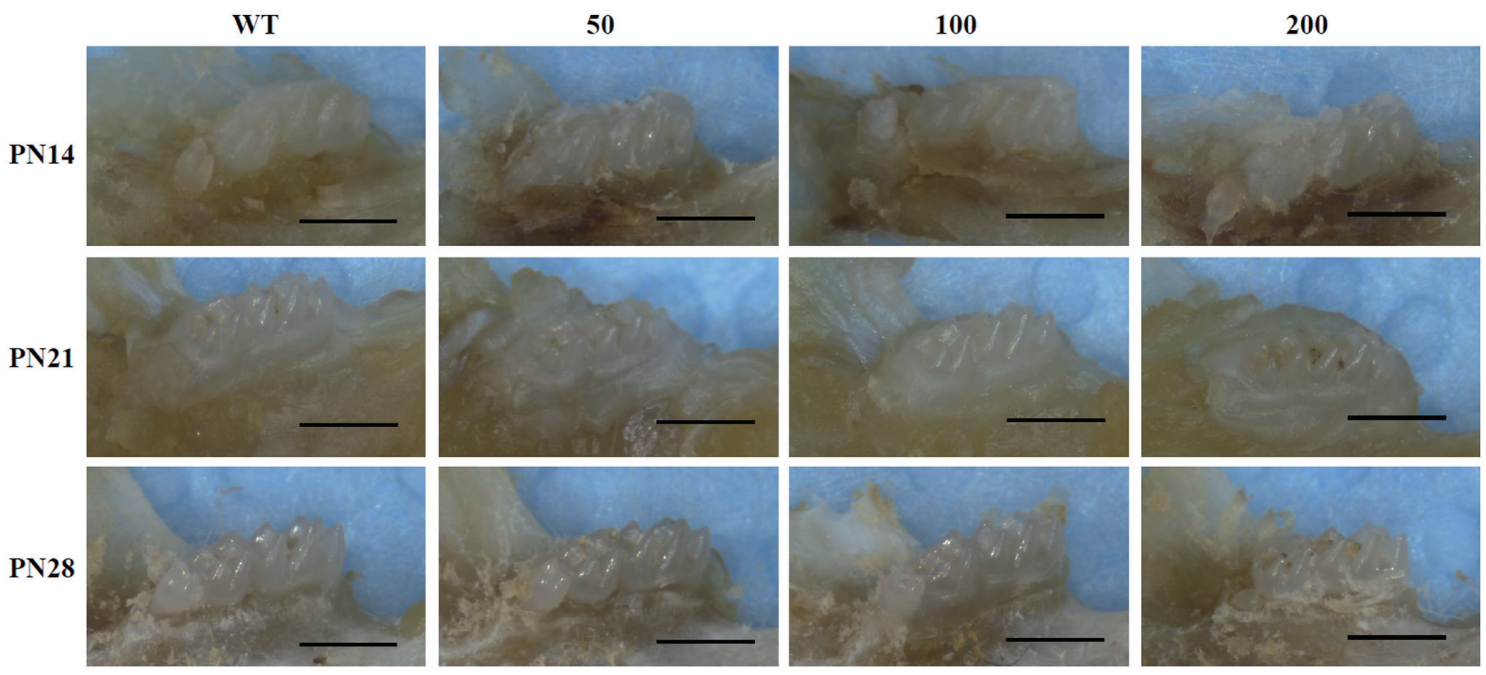

Figure 2. Effects of CPA on tooth eruption and crown shape. Representative macroscopic images of removed molars at PN 14, 21, and 28. WT: wild type (control). Scale bars: $250 \mu \mathrm{m}$

ing alopecia and weight loss in mice, but it was not lethal.

\section{Effects of CPA treatment on tooth eruption and crown shape}

We examined whether CPA treatment influenced developmental abnormalities, including tooth eruption and crown shape. Fig. 2 shows macroscopic images of the removed mandibles. At PN 14, a complete eruption of the first molar was observed in all groups. There were no differences in crown shape morphology of the molar among mice in the treated or control groups. These findings indicate that the CPA treatment did not effect tooth eruption or lead to abnormal crown shapes.

\section{CPA treatment induces arrested root development}

To examine the effect of CPA on the root development, we compared the length of the medial first molar roots between the control and CPA-treated groups (Figs. 3A and B). Root length increased chronologically in all groups and did not differ significantly among control, $50 \mathrm{mg} /$ 
A

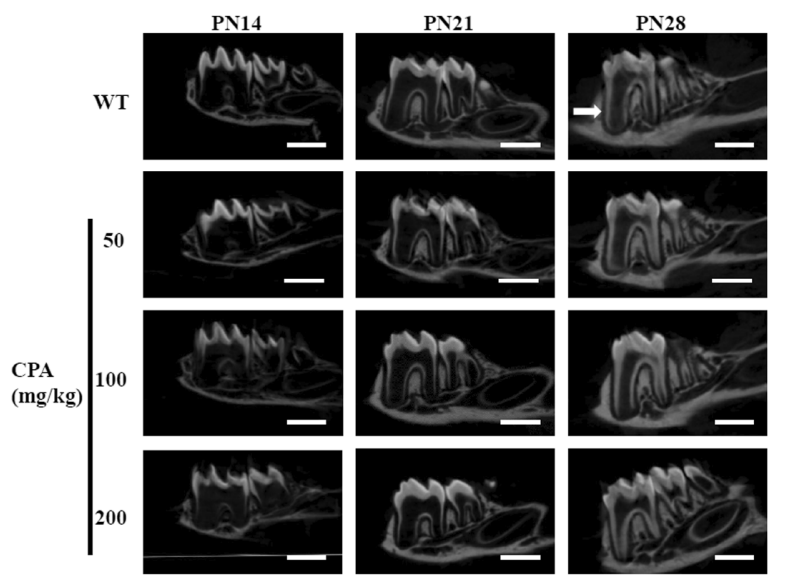

B

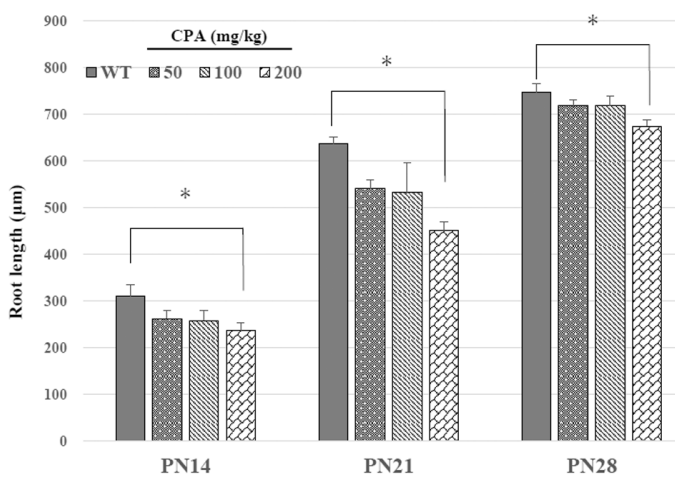

Figure 3. Chronological changes in the length of the medial roots of the mandibular first molars from mice treated with or without CPA at PN 14, 21, and 28. A: Three-dimensional (3D) structure of the unilateral mandibles. Root length was obtained from micro-CT images of the medial roots (arrow) of mandibular first molars through computed 3D reconstruction measured from the cementoenamel junction to the root apex. B: Changes in the medial root length at PN 14, 21, and 28. The results are expressed as the mean \pm SD. *Statistically significant difference compared with the control $(\mathrm{p}<0.05)$. WT: wild type (control). Scale bars: $250 \mu \mathrm{m}$

A

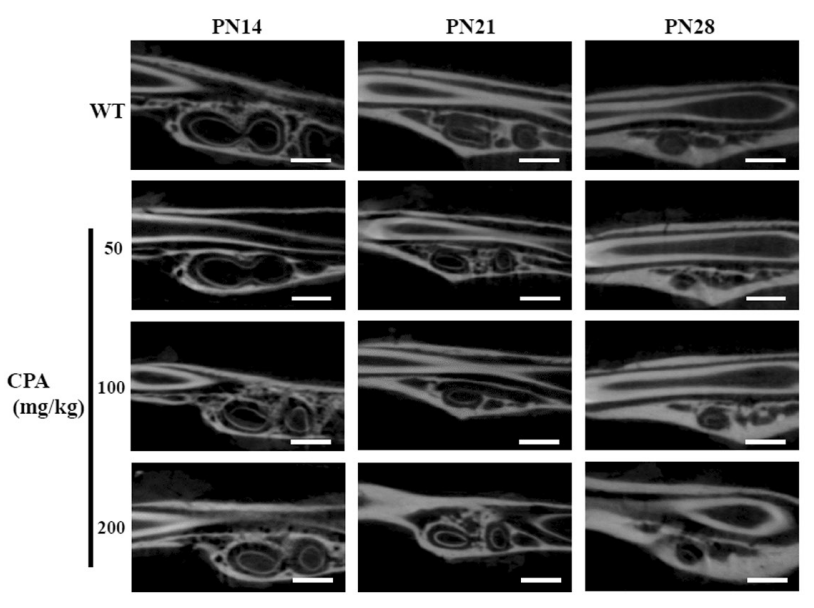

B

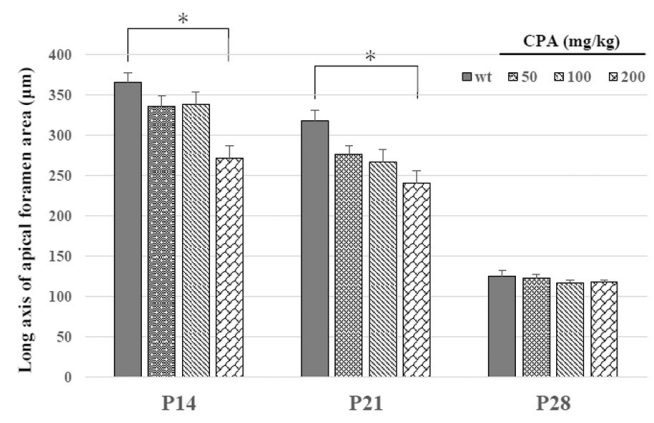

P14

P21

P28

Figure 4. Chronological changes of the apical foramina in the medial roots of the mandibular first molars from mice treated with or without CPA. A: Micro-CT images of apical foramen areas of the extracted mandibular first molars through computed 3D reconstruction. B: Changes in the sizes of the apical foramina in the medial roots at PN14, 21, and 28 . The results are expressed as the mean \pm SD. $*$ Statistically significant difference compared with the control $(\mathrm{p}<0.05)$. WT: wild type (control), Scale bars: $250 \mu \mathrm{m}$

$\mathrm{kg}$ CPA, and $100 \mathrm{mg} / \mathrm{kg}$ CPA groups, whereas there was significantly reduced root length in the $200 \mathrm{mg} / \mathrm{kg} \mathrm{CPA}$ group at all time. Indeed, the mean root length in the $200 \mathrm{mg} / \mathrm{kg}$ CPA group was approximately $65 \%$ of that in the control group at PN 21.

We next examined the effect of CPA on apical foramina. Fig. 4 shows changes in the mean apical foramen area in the medial roots of the mandibular first molars from PN 14 to PN 28. The apical foramen from all groups decreased in size according to age. In particular, the apical foramen area from mice treated with $200 \mathrm{mg} / \mathrm{kg}$ CPA was significantly smaller than those from control mice at PN 14 and PN 21 (p < 0.05). At PN 28, all groups showed almost the same size in the apical foramen area. These findings indicate that mice treated with $200 \mathrm{mg} / \mathrm{kg}$ CPA showed an early completion of root development, compared with untreated mice and those treated with low dosages of CPA.

\section{Histological analysis of root formation in the mandibular molars} treated with or without $\mathrm{CPA}$

To elucidate the effects of CPA on the stage of root formation, we examined H\&E-stained sections of the developing root in the first molars of the mandible at PN 14, 21, and 28. Fig. 5 shows the histological changes in the periapical region of the medial root at each time point. In all groups, the medial roots showed a broad root canal and wide opening of the root apices at PN 14. At PN 21, epithelial diaphragm appeared in the root apex of the $200 \mathrm{mg} / \mathrm{kg}$ group, whereas the other groups became open. The root canals near the apex became narrow in all groups at PN 28. Particularly, the root canals in the CPA groups were markedly narrower than those in the control group. A closed apex was observed histologically in the $200 \mathrm{mg} / \mathrm{kg}$ group. The histological findings, combined with the micro-CT results, suggest that the $200 \mathrm{mg} / \mathrm{kg}$ CPA group shows an early apical closure in the root of the molars. 


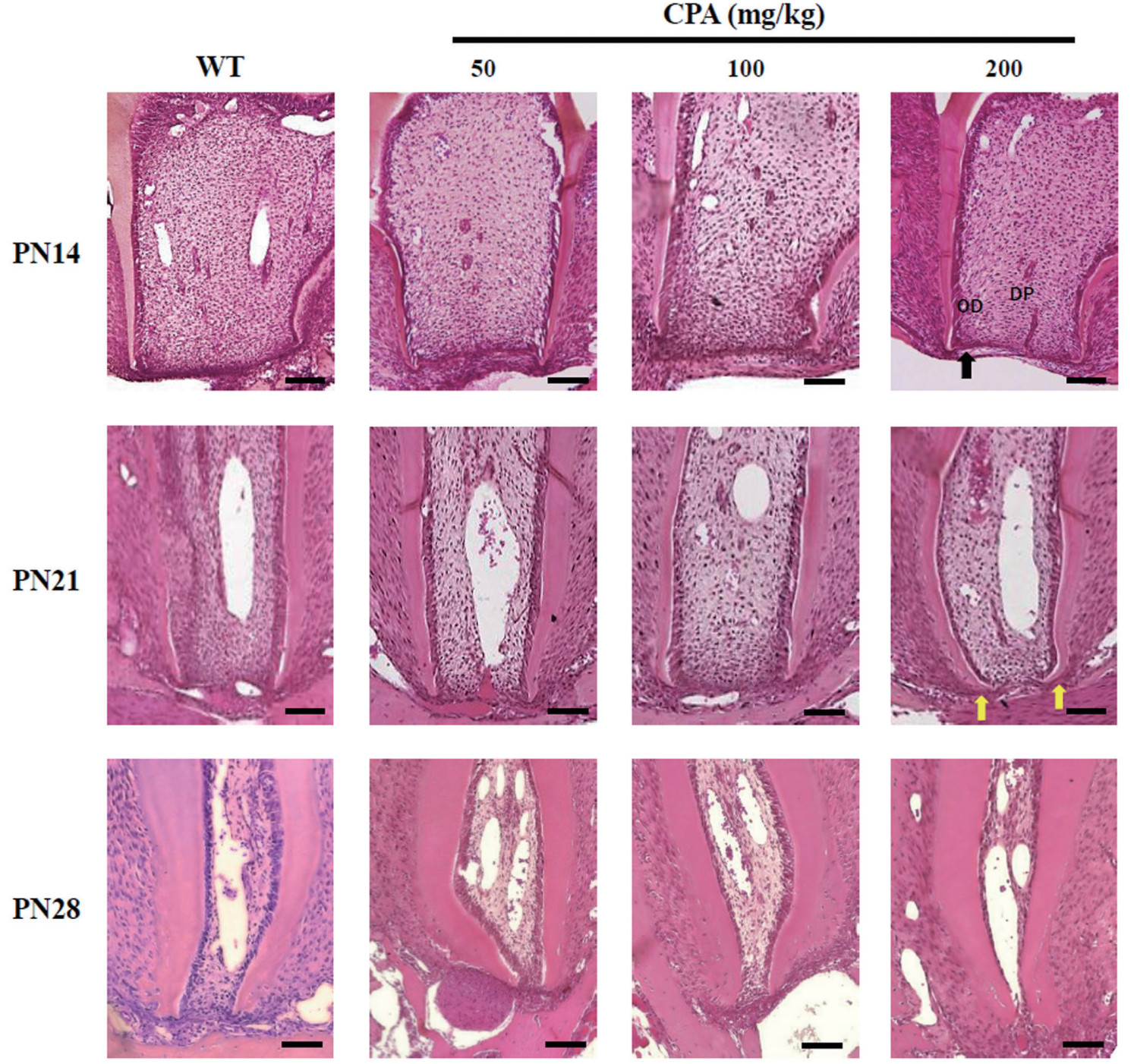

Figure 5. Histological changes in the root apices of the mandibular first molars. H\&E staining shows the medial root of the molars in the control and CPA groups at PN 14, 21, and 28. WT: wild type (control), DP: dental pulp, OD: odontoblasts, Black arrow: HERS (Hertwig's epithelial root sheath), Yellow arrows: epithelial diaphragm, Scale bars: $100 \mu \mathrm{m}$

\section{Effects of CPA on HERSO1 a cells}

To elucidate in vitro effects of CPA on HERS cells, we cultured the HERS cell line (HERS01a cells). In the phase contrast images of HERS01a cells, cell damage was observed in the HERS01a cells treated with CPA, irrespective of CPA concentration (Fig. 6A). Furthermore, we examined the effects of CPA on cell viability of HERS01a cells using a trypan blue dye exclusion assay. The assay showed a significant decrease in the viability of HERS01a cells treated with $0.36 \mu \mathrm{M}(82.0 \pm$ $3.00)$ and $0.72 \mu \mathrm{M}(68.3 \pm 3.51)$ of $\mathrm{CPA}$, compared with the control $(P$ $=0.045$ vs. $0.36 \mu \mathrm{M} ; P=0.0003$ vs. $0.72 \mu \mathrm{M})$ and $0.18 \mu \mathrm{M}$ CPA groups (Fig. 6B). These findings indicate that CPA treatment can affect cell viability of HERS01a cells in a dose-dependent manner.

\section{Changes in epithelial and mesenchymal characteristics of HERSO1a cells treated with $\mathrm{CPA}$}

We examined whether characteristics of HERS cells changed due to CPA treatment. Expression of E-cadherin, which is known as an epithelial marker in HERS cells, gradually decreased with increased CPA concentration. A marked decrease in E-cadherin expression was observed in the $0.72 \mu \mathrm{M}$ CPA group (Fig. 7A), compared with the other groups, indicating that the CPA treatment can induce a loss of cell-cell adhesion in the HERS cells. In contrast, $\mathrm{N}$-cadherin expression, which is a reliable marker for mesenchymal components, was expressed in HERS cells treated with a high concentration of CPA (Fig. 7A), suggesting that cells showing impaired cell-cell contact due to downregulated expression of E-cadherin may be susceptible to increased $\mathrm{N}$-cadherin expression.

We next evaluated immunocytochemical expression of both E-cadherin and N-cadherin in HERS cells treated with or without CPA, to elucidate the effect of CPA on their cellular behavior (Fig. 7B). In the control group, HERS cells that showed a cobblestone-like arrangement, revealed membrane-bound expression of E-cadherin, whereas no N-cadherin expression was observed. Membrane-bound expression of E-cadherin was gradually decreased in HERS cells with increases in CPA concentration. In particular, E-cadherin expression nearly disappeared in most HERS cells treated with $0.72 \mu \mathrm{M}$ CPA. Conversely, significant expression of N-cadherin was observed in cells of the 0.36 and $0.72 \mu \mathrm{M}$ CPA groups. These findings suggest that CPA treatment, particularly at higher concentrations, can initiate an EMT-like phenomenon in the 
A
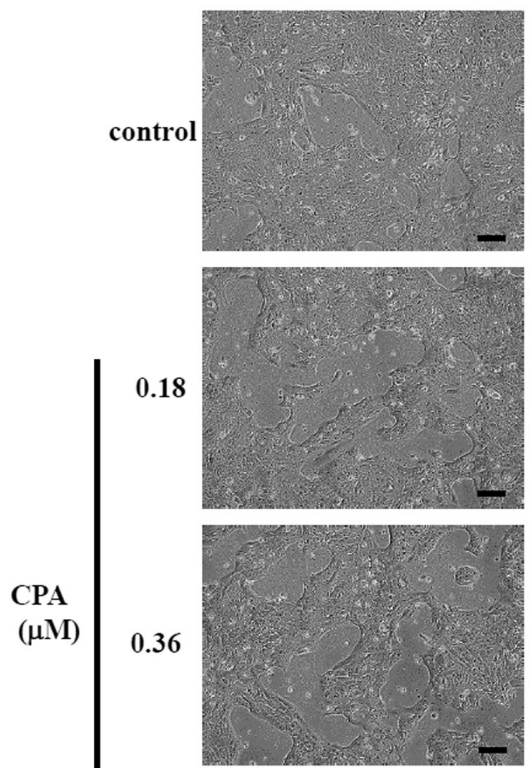

0.72

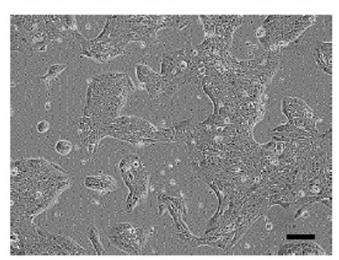

B

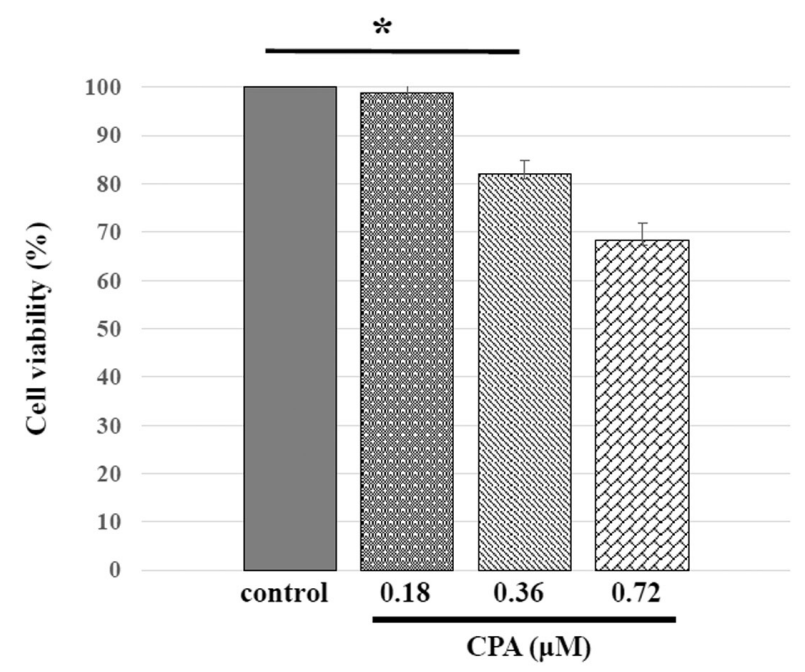

Figure 6. In vitro effects of CPA treatment on HERS01a cells. A: Phase contrast images of HERS01a cells. B: Cell viability was determined using trypan blue exclusion. Viability of HERS01a cells exposed to different concentrations $(0.18,0.36$, and $0.72 \mu \mathrm{M})$ of CPA for $48 \mathrm{~h}$. Results are presented as the percentage of the trypan blue exclusion in untreated cells (control). Data represent mean values \pm SD in quadruplicate. ${ }^{*} \mathrm{p}<0.05$ vs. the $0.72 \mu \mathrm{M}$ group. There is no significant difference in groups of columns jointed to horizontal bar. Scale bars: $100 \mu \mathrm{m}$

A
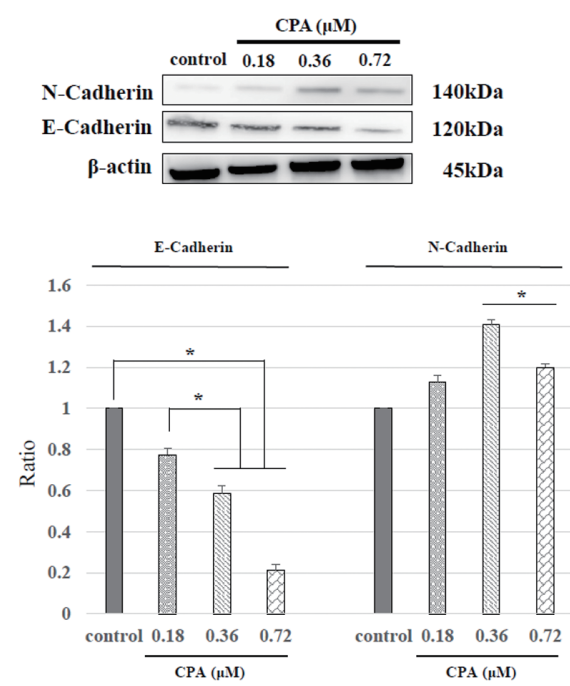

B

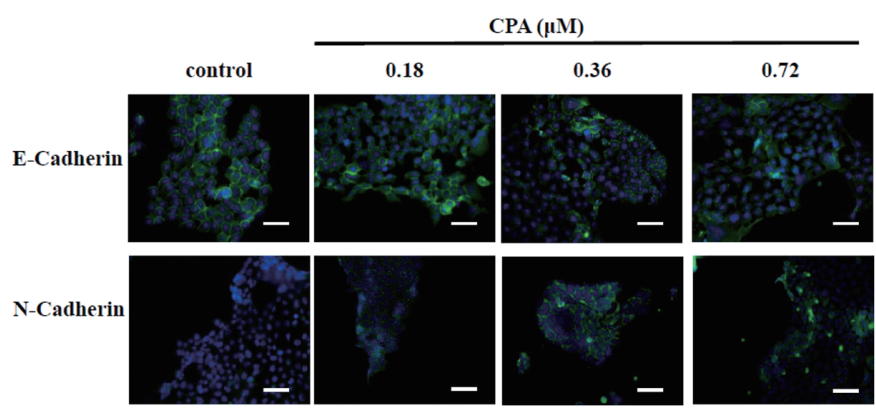

Figure 7. Effects of CPA treatment on expression of epithelial and mesenchymal markers in HERS01a cells. A: Protein expression of E-cadherin, N-cadherin, and beta-actin in HERS01a cells treated with or without CPA. Quantification of proteins was performed using the NIH ImageJ software, and values shown are fold increases normalized to those of b-actin as a control. Similar results were obtained in three independent experiments. Data represent mean values \pm SD in quadruplicate. ${ }^{*} \mathrm{p}<0.05$ vs. the control and $0.18 \mu \mathrm{M}$ groups. There is no significant difference in groups of columns jointed to horizontal bar.

B: Immunofluorescence (green) of E-cadherin and N-cadherin in the cells treated with or without CPA. 4', 6-Diamidino-2-phenylindole (DPAI) was used to label the nuclei (blue). Scale bars: $50 \mu \mathrm{m}$ 
HERS cells.

\section{Discussion}

There is strong evidence that supports an association between chemotherapy and dental developmental abnormalities, including dental genesis, dental hypoplasia, root stunting, and enamel hypoplasia. Among those abnormalities, arrested root development, such as short V-shaped roots and early apical closure are relatively common findings in survivors of childhood cancer ${ }^{22-24}$. To elucidate the adverse effects of $\mathrm{CPA}$ on dental root formation, we analyzed morphological changes in the molar roots of mice using CT and histological images. Furthermore, in vitro cell culture studies investigated the mechanism underlying the cellular effects of the CPA treatment on HERS01a cells. Our in vivo and in vitro results showed that CPA treatment at the early stages of root formation can induce impaired root development, via a disturbance in epithelial components of HERS cells.

Treatment with higher dosages of CPA led to the systemic disorders, such as weight and hair losses. Both weight loss and alopecia are the most common side effects of chemotherapy. In this study mice treated with $200 \mathrm{mg} / \mathrm{kg}$ CPA revealed remarkable side effects. Although the 200 $\mathrm{mg} / \mathrm{kg}$ dose is higher than that used in low- or standard-risk protocols postulated by the Tokyo Children's Cancer Study Groups, high-dosages of CPA administration is occasionally used for clinical therapy in certain patient groups, such as high-risk or extremely high-risk groups. CPA treatment induces these disorders, because its cytotoxic effects rapidly attack nonspecifically dividing cells in the body ${ }^{25}$. Particularly, separation of hair at the bulb and hair shaft breakage due to impaired proliferation of the hair follicle matrix keratinocytes can cause chemotherapy-induced alopecia ${ }^{26}$. Furthermore, mice with these systemic disorders showed impairment dental development.

Our macroscopic and CT images showed no significant changes in stages of tooth eruption or crown morphology among all groups. These findings were unexpected, because clinical and experimental studies reported that CPA can induce disturbances in tooth development ${ }^{27,28)}$. As the timing of CPA administration did not influence the development stages of tooth crown formation, no morphological changes in the crown were observed in the molars, irrespective of CPA dosages. These findings are consistent with a recent report that nondividing columnar odontoblasts and ameloblasts in the mice incisor were not affected by CPA administration ${ }^{29}$. Delayed tooth eruption is well-known as a long-term side effects of chemotherapy ${ }^{27)}$. Tooth eruption is a complex and tightly regulated process that is divided into five stages; preeruptive movements, intraosseous stage, mucosal penetration, preocclusal and post occlusal stages ${ }^{30)}$. Delayed tooth eruption is caused by impairment of the pre-eruptive movement in eruptive stages. CPA administration did not influence the timing of pre-eruptive movement stage during the process of tooth eruption.

High-dosages of CPA administration can induce the arrested root development, when compared with the control group and with mice treated with low dosages of CPA. In this study, the length of the tooth root was significantly shorter than that of the other groups. Recent studies reported that CPA administration can result in decreased root length in the first molar of rat mandible, indicating that the effects of CPA are correlated with the developmental stage of the tooth ${ }^{27,28)}$. Consistent with recent report that demonstrated inhibition of root growth in a dose-dependent manner of CPA treatment ${ }^{24,31)}$, we revealed that the high dosage of CPA administration developed decreased root length in the first molars of the mandibles. Induction of the decreased root length may be explained by the general effects of CPA administration on arrested mitotic activity in the root proliferation zone, consisting of HERS or odontoblasts, as it is believed that HERS is important for root formation and determines the root shape and size ${ }^{17}$. Additional abnormality of CPA-induced disturbances in root development induces early closure of the apical foramen. In our histological analysis, the cervical loop structure was shown in the apical region of the tooth from mice treated with high dosages of CPA at PN 14. Along with the appearance of the cervical loop structure, the direction of HERS cells to the central portion of the apex. The change in direction of HERS cells leads to the closure of the apical foramen. The apical closure of the tooth implies complete root forma$\operatorname{tion}^{32}$. A recent paper speculated that premature closure of apices led to decreases in tooth length ${ }^{9)}$. In the high-dosage group, the early closure of the apical foramen induced the completion of the root development and contributed to the short root length. These findings suggest that the inducible change in direction of HERS by CPA administration may lead to short root malformation.

In this study, the results of cell culture experiments, using HERS01a cells, revealed that CPA treatment induced dysregulation of HERS cell components. Significant changes were observed in HERS cells treated with high-dose CPA, and alterations in cellular components were dose-dependent. Our western blotting and immunocytochemical analyses confirmed that HERS cells lost E-cadherin expression, while N-cadherin expression was upregulated. This phenomenon of cadherin switch is known as the $\mathrm{EMT}^{33,34)}$. Recent studies represented that TGF- $\beta$ stimulation could induce EMT in HERS cells ${ }^{35,36}$. Therefore, we postulate that CPA treatment can also facilitate EMT-like movement in the HERS cells. Downregulated expression of E-cadherin induced by CPA treatment is responsible for the degradation of cell-cell adhesion of HERS. From these findings, we speculate that HERS fragmentation induced by an EMT-like phenomenon leads to the formation of cervical loop structure in the apical portion of the root. In contrast to normal root development that progressed due to continuous elongation of the cervical loop, the CPA-induced cervical loop may prompt an early closure of the apical foramen, resulting in a shorter root.

Our potential limitation to this study is the lack of direct evidence to examine immunohistochemical expression of the EMT-related molecules in the HERS cells of the tooth root in the mandibles of CPA-treated mice. Further studies are warranted that elucidate the immunohistochemical changes in the EMT-related molecules in HERS cells of CPA-treated mice. Moreover, we also must investigate the molecular mechanisms for the induction of the EMT in CPA-treated HERS cells.

In conclusion, the findings of this study shed additional light on the effects of CPA on root development abnormalities. Administration of high-dose CPA leads to shorter roots that are associated with the early closure of the apical foramen. Furthermore, CPA treatment prompts an EMT-like phenomenon, suggesting that the EMT-induced formation of the cervical loop structure may cause the apical foramen closure and lead to shorter roots.

\section{Acknowledgments}

We would like to thank Prof. Hidemitsu Harada for kindly providing us with HERS01a cells and Enago (Academic Proofreading Service; www.enago.jp). This work was supported by JSPS KAKENHI (grant numbers: $18 \mathrm{~K} 09567$ to JO and 17K17344 to MT-N) and the Private University Research Branding Project.

\section{Conflict of Interest}

The authors have no competing interest to declare. 


\section{References}

1. Maeda M. Late effects of childhood cancer: life-threatening issues. J Nippon Med Sch 75: 320-324, 2008

2. Mertens AC, Yong J, Dietz AC, Kreiter E, Yasui Y, Bleyer A, Armstrong GT, Robison LL and Wasilewski-Masker K. Conditional survival in pediatric malignancies: analysis of data from the Childhood Cancer Survivor Study and the Surveillance, Epidemiology, and End Results Program. Cancer 121: 1108-1117, 2015

3. Hsieh SG, Hibbert S, Shaw P, Ahern V and Arora M. Association of cyclophosphamide use with dental developmental defects and salivary gland dysfunction in recipients of childhood antineoplastic therapy. Cancer 117: 2219-2227, 2011

4. Ishida Y, Sakamoto N, Kamibeppu K, Kakee N, Iwai T, Ozono S, Maeta N, Okamura J, Asami K, Inada H, Honda M and Horibe K. Late effects and quality of life of childhood cancer survivors: Part 2. Impact of radiotherapy. Int J Hematol 92: 95-104, 2010

5. Effinger KE, Migliorati CA, Hudson MM, McMullen KP, Kaste SC, Ruble K, Guicher GM, Shah AJ and Castellino SM. Oral and dental late effects in survivors of childhood cancer: a Children's Oncology Group report. Support Care Cancer 22: 2009-2019, 2014

6. Goho C. Chemoradiation therapy: effect on dental development. Pediatr Dent 15: 6-12, 1993

7. Cubukcu CE, Sevinir B and Ercan I. Disturbed dental development of permanent teeth in children with solid tumors and lymphomas. Pediatr Blood Cancer 58: 80-84, 2012

8. Duggal MS. Root surface areas in long-term survivors of childhood cancer. Oral Oncol 39: 178-183, 2003

9. Purdell-Lewis DJ, Stalman MS, Leeuw JA, Humphrey GB and Kalsbeek H. Long term results of chemotherapy on the developing dentition: caries risk and developmental aspects. Community Dent Oral Epidemiol 16: 68-71, 1988

10. Wang X-P. Tooth eruption without roots. J Dent Res 92: 212-214, 2012

11. Gowgiel JM. Observations on the phenomena of tooth eruption. J Dent Res 46: 1325-1330, 1967

12. Carl W and Wood R. Effects of radiation on the developing dentition and supporting bone. J Am Dent Assoc 101: 646-648, 1980

13. Brin I, Zilberman Y, Galili D and Fuks A. Eruption of rootless teeth in congenital renal disease. Oral Surg Oral Med Oral Pathol 60: 6164. 1985

14. Kalk WW, Batenburg RH and Vissink A. Dentin dysplasia type I: five cases within one family. Oral Surg Oral Med Oral Pathol Oral Radiol Endod. 86: 175-178, 1998

15. Nirmala SV, Sivakumar N and Usha K. Dentin dysplasia type I with pyogenic granuloma in a 12-year-old girl. J Indian Soc Pedod Prev Dent. 27: 131-134, 2009

16. Orban BJ. Orban's oral histology and embryology. Mosby, St. Louis, 1980

17. Ten Cate AR. The role of epithelium in the development, structure and function of the tissues of tooth support. Oral Dis 2: 55-62, 1996

18. Huang X, Bringas P, Jr., Slavkin HC and Chai Y. Fate of HERS during tooth root development. Develop Biol 334: 22-30, 2009

19. Luan X, Ito Y and Diekwisch TGH. Evolution and development of Hertwig's epithelial root sheath. Dev Dyn 235: 1167-1180, 2006
20. Luder HU. Malformations of the tooth root in humans. Front Physiol 6: 307, 2015

21. Akimoto T, Fujiwara N, Kagiya T, Otsu K, Ishizeki K and Harada H. Establishment of Hertwig's epithelial root sheath cell line from cells involved in epithelial-mesenchymal transition. Biochem Biophys Res Commun 404: 308-312, 2011

22. Pedersen LB, Clausen N, Schroder H, Schmidt M and Poulsen S. Microdontia and hypodontia of premolars and permanent molars in childhood cancer survivors after chemotherapy. Int J Paediatr Dent 22: 239-243, 2012

23. Minicucci EM, Lopes LF and Crocci AJ. Dental abnormalities in children after chemotherapy treatment for acute lymphoid leukemia. Leuk Res 27: 45-50, 2003

24. Lopes NN, Petrilli AS, Caran EM, Franca CM, Chilvarquer I and Lederman H. Dental abnormalities in children submitted to antineoplastic therapy. J Dent Child (Chic) 73: 140-145, 2006

25. de Jonge ME, Huitema AD, Rodenhuis S and Beijnen JH. Clinical pharmacokinetics of cyclophosphamide. Clin Pharmacokinet 44: 1135-1164, 2005

26. Paus R, Handjiski B, Eichmuller S and Czarnetzki BM. Chemotherapy-induced alopecia in mice. Induction by cyclophosphamide, inhibition by cyclosporine A, and modulation by dexamethasone. Am J Pathol 144: 719-734, 1994

27. Nasman M and Hammarstrom L. Influence of the antineoplastic agent cyclophosphamide on dental development in rat molars. Acta Odontol Scand 54: 287-294, 1996

28. Nasman M, Hultenby K and Forsberg CM. A scanning electron microscopy study of disturbances in the developing rat molar induced by cyclophosphamide. Acta Odontol Scand 55: 186-191, 1997

29. Anton E. Ultrastructural study of the effect of cyclophosphamide on the growth area of incisor teeth of DBA/2 and C57BL/6 mice. Int J Exp Pathol 77: 83-88, 1996

30. Nolla CM. The developmnet of the human dentition. ASDC J Dent Child 27: 254-266, 1960

31. Kawakami T, Nakamura Y and Karibe H. Cyclophosphamide inhibits root development of molar teeth in growing mice. Odontology 103: 143-151, 2015

32. Thomas HF. Root formation. Int J Dev Biol 39: 231-237, 1995

33. Wang Y, Shi J, Chai K, Ying X and Zhou BP. The role of snail in EMT and tumorigenesis. Curr Cancer Drug Targets 13: 963-972, 2013

34. Katsuno Y, Lamouille S and Derynck R. TGF-b signaling and epithelial-mesenchymal transition in cancer progression. Curr Opin Oncol 25: 76-84, 2013

35. Itaya S, Oka K, Ogata K, Tamura S, Kira-Tatsuoka M, Fujiwara N, Otsu K, Tsuruga E, Ozaki M and Harada H. Hertwig's epithelial root sheath cells contribute to formation of periodontal ligament through epithelial-mesenchymal transition by TGF-b. Biomed Res 38: 61-69, 2017

36. Chen J, Chen G, Yan Z, Guo Y, Yu M, Feng L, Jiang Z, Guo W and Tian W. TGF-b1 and FGF2 stimulate the epithelial-mesenchymal transition of HERS cells through a MEK-dependent mechanism. J Cell Physiol 229: 1647-1659, 2014 